REVIEW

\title{
Melatonin supplementation for severe and intractable sleep disturbance in young people with genetically determined developmental disabilities: short review and commentary
}

\section{J Turk}

J Med Genet 2003;40:793-796

Serious childhood developmental disabilities are common, and are debilitating for the individual and their family. Increasingly these are being shown to have genetic bases. Associated challenging behaviours are frequent, one of the commonest being severe and intractable sleep disturbance. This is associated with daytime behaviour problems, poor developmental and academic progress, and substantial familial psychopathology. Social and behavioural approaches ("sleep hygiene" measures) have, to an extent, revolutionised management; however, many individuals remain unresponsive. Modern medications therefore play increasingly important complementary roles in conjunction with psychological, educational, and social strategies. This paper reviews evidence for the frequency and severity of sleep disturbance in children and young people with severe intractable neurodevelopmental disabilities. The potential benefits of judicious and carefully monitored use of medication are described, with a focus on the importance of melatonin as a sleep inducer. It is concluded that melatonin is a potentially useful and safe adjunct to psychological and social approaches for severe sleep disturbance in this client group.
Correspondence to: Dr J Turk, Department of Clinical Developmental Sciences, St. George's Hospital Medical School, Cranmer Terrace, London SW17 ORE, UK j.turk@sghms.ac.uk
$M$ elatonin $\quad(N$-acetyl-5-methoxytryptamine) is a naturally occurring indole serotonin derivative, secreted by the pineal gland in response to falling light levels as picked up by the eyes. Melatonin is synthesised in the pineal gland from tryptophan as shown below:

Tryptophan $\rightarrow$ serotonin $\rightarrow N$-acetylserotonin $\rightarrow$ melatonin ( $N$-acetyl-5methoxytryptamine).

Nerve impulses are conveyed from retina to pineal gland by a well documented neuronal pathway:

Retina $\rightarrow$ suprachiasmatic nucleus of hypothalamus $\rightarrow$ reticular system $\rightarrow$ spinal cord $\rightarrow$ cervical ganglia $\rightarrow$ postganglionic sympathetic fibres $\rightarrow$ pineal gland.
Melatonin has acquired publicity in relation to its use for jet lag. Less attention has been paid to its potential in treating intractable sleep disturbance in young people with developmental disabilities. Such problems are common, ${ }^{1}$ particularly in children with multiple neurological disabilities, ${ }^{2}$ and often fail to respond to behavioural and social interventions.

\section{SLEEP DIFFICULTIES IN CHILDREN AND YOUNG PEOPLE WITH INTELLECTUAL DISABILITY}

Quine $^{2}$ found sleep problems to be extremely frequent in young people with severe learning difficulties (mental handicap); $51 \%$ of 200 children had settling problems, $67 \%$ had waking problems, and $32 \%$ of parents reported rarely getting enough sleep. Sleep problems were associated with poor communication, academic and self help skills, incontinence, daytime behavioural problems, and epilepsy. Sleep problems also related to parental stress and irritability, and familial impact generally. Problems persisted over time and were related to diagnoses of cerebral palsy and other named conditions, with the exception of Down's syndrome. This finding, along with the association with epilepsy, supports the hypothesis of strong neurological components in sleep disorder in young people with developmental disabilities. This contrasts with primarily social determinants of sleep (zeitgebers) in people with average intellectual abilities. Quine found no relationships between sleep problems and family variables such as social class, income, family composition, or housing tenure, and concluded that families containing children with irregular sleeping problems are often families with many other problems of family functioning. However, direction of causality in this relationship is often unclear, and can be misattributed, with family dysfunctionality and psychopathology being seen as the cause of the child's sleep disturbance, rather than its consequence.

Intellectual disability itself predisposes to marked sleep difficulties. These include irregular sleep/wake patterns, diminished responses to external stimuli, prolongation of sleep/wake cycle, diminished rapid eye movement sleep,

Abbreviations: ADHD, attention deficit hyperactivity disorder; aMT6, sulphatoxymelatonin 
increased frequency of night time waking, and delays in returning to sleep. ${ }^{3}$ Certain genetic causes of intellectual disability appear to have specific relationships with particular sleep problems. In Down's syndrome, despite sleep problems being rarer than in other children with intellectual disability, they are still far more common than in the general population, and contribute to parental stress, poor parentchild relationships, and family cohesion issues. ${ }^{4}$ Obstructive sleep apnoea is also commonly reported. ${ }^{5}$ Individuals with Prader-Willi syndrome may also have obstructive sleep apnoea, as well as initial insomnia, repeated night waking, and increased nocturnal sleep duration. ${ }^{6}$ Marked obesity resulting from hyperphagia is a complicating factor, yet efforts at behavioural approaches to dietary control confirm just how entrenched and compulsive is eating behaviour. Excessive daytime sleepiness with increased behavioural disturbance, and sleep onset rapid eye movement periods have been reported, yet it has not proved possible to link these problems with particular genetic variants or a single genetic model. ${ }^{7}$ Snoring and early morning waking also occur significantly more frequently. ${ }^{8}$ Various authors have attributed these problems to combinations of extreme obesity, upper airway obstruction, and hypothalamic dysfunction.

Individuals with mucopolysaccharidoses have increased rates of obstructive airways and sleep disordered breathing, related to structural/anatomical abnormalities. ${ }^{9}$ Milder skeletal abnormalities in Sanfillipo syndrome make obstructive sleep apnoea less common. However, Bax \& Colville ${ }^{10}$ report high rates of settling and waking problems, short sleep duration, and frequent hyperkinetic tendencies in this subgroup. Gould et $a l^{11}$ found boys with fragile X syndrome to have greater variability in total sleep time and difficulty in sleep maintenance compared with age matched boys of average intelligence. However, failure to match for intellectual level makes findings difficult to interpret. The authors also noted elevated nocturnal and daytime melatonin levels in the fragile $\mathrm{X}$ participants.

Possibly the greatest interest in sleep disturbance of genetic aetiology has been in Smith-Magenis syndrome, caused by interstitial chromosomal deletion 17p11.2. Individuals with this syndrome have been documented as being prone to intellectual disability, attention deficits, and overactivity, severe self injury in the form of finger and toe nail pulling (onychotillomania) and insertion of objects into bodily orifices (polyembolokoilomania), and social and communicatory difficulties. ${ }^{12}$ They may also demonstrate a characteristic intermittent spasmodic upper body squeeze. ${ }^{13}$ In addition, individuals have a phase shift of their circadian rhythm of melatonin with paradoxical diurnal secretion. Sleep problems have been reported in $5-100 \%$ in samples studied. ${ }^{14}$ Problems include difficulties falling asleep, shortened sleep cycles, frequent and prolonged nocturnal wakings, excessive daytime sleepiness, daytime napping, snoring, and bedwetting.

\section{MELATONIN}

Interest in the therapeutic use of melatonin for sleep disturbance in young people increased following publication of the seminal paper from Jan et al. ${ }^{15}$ This report described benefits of melatonin supplementation in improving sleep in 15 children, most of whom had multiple neurological disabilities. The authors commented on "significant health, behavioural, and social benefits of treatment". However, responses were not always complete and varied considerably depending on type of sleep disturbance. In addition, the study did not fulfil strict "double blind" criteria in terms of experimenter and participant awareness of whether active melatonin or placebo was being received.
The first successful report of treatment of sleep disturbance with melatonin was by Miles et al. ${ }^{16} \mathrm{~A}$ blind man with sleep difficulties showed considerable improvement following commencement of oral melatonin supplementation. The authors speculated on the relevance of the individual's visual impairment to postulated disruption in endogenous melatonin secretion. Palm et al ${ }^{17}$ published a case report on a multiply disabled, visually impaired 9 year old child with ocular blindness whose sleep was "normalised" by melatonin $0.5 \mathrm{mg}$ nightly, prescribed on a regular daily basis.

There have been numerous reports of the value of melatonin in inducing sleep in other groups at risk of insomnia. For example, usefulness has been proposed for those working shifts, ${ }^{18}$ or suffering jet lag, ${ }^{19}$ chronic insomnia, ${ }^{20}$ or delayed sleep phase syndrome. ${ }^{21}$ Jan et al ${ }^{15}$ found beneficial effects of melatonin in their 15 children ( 13 male) with severe chronic sleep disorders who had failed to respond to conventional psychological and social management. They also suggested occasional improvements in attention span, school performance, self injury, and even epilepsy control. The authors hypothesised particular difficulty in formulating appropriate cerebral signals for melatonin release in multiply disabled children. However, their study has a number of limitations, including the following:

- Six of 15 participants were given melatonin immediately after baseline assessments owing to "family crises".

- The degree of visual impairment varied: nine had ocular or cortical visual impairment while the remainder had good visual acuity.

- Reports of behavioural change were largely anecdotal and unstructured.

- The nature and degree of reported improvement varied substantially.

- The type of sleep disturbance varied: nine fragmented sleep patterns; three delayed sleep onset; and three "nonspecific sleep disturbance of unclear aetiology".

- There was no structured follow up.

In a subsequent letter, Jan \& Espezel ${ }^{22}$ reported full or partial correction of sleep/wake cycle disturbance in almost 90 children with a range of developmental disabilities, neurological disorders and chromosomal anomalies. The importance of continuing strict environmental sleep structuring was emphasised. Side effects and tolerance were not noted.

More recently, Pillar et $a^{23}$ investigated the biochemistry of chronic sleep disturbance and its treatment with melatonin further. In a single case study of a 13 year old girl with "psychomotor retardation" they found urinary sulphatoxymelatonin (aMT6) concentrations to be abnormally low with no diurnal variation. Evening melatonin increased nocturnal urinary aMT6 concentrations and improved sleep/wake pattern.

Other lines of research have produced findings consistent with melatonin being useful for sleep problems in at risk groups. Some children not responding to hypnotics and sedatives have been reported as responding to light treatment, which modifies melatonin secretion. ${ }^{24}$ Interestingly, light treatment has also found to be beneficial for adults of normal intelligence who have seasonal affective disorder. Both mood and sleep quality and quantity have been reported as improving. It is worth noting in this respect that serotonin, the central neurotransmitter known to be intimately involved in human mood regulation, is an intermediary in the melatonin biochemical pathway.

Some research groups have explored sleep disturbance and melatonin in specific genetic disorders. Benefits have been reported for seven individuals with tuberous sclerosis and 
severe sleep problems. ${ }^{25}$ There was a small but significant improvement in total sleep time of a half hour, but only a non-significant improvement in sleep onset time. Scrutinising the data of O'Callaghan et al suggests two participant groups: responders and non-responders. The important clinical and experimental issue is therefore whether "responders" do so consistently while showing little if any improvement on placebo. This indicates the need for two stage trials: firstly, identification of responders, and secondly, confirmation of consistency of their response. McArthur \& Budden ${ }^{26}$ reported improved sleep onset latency, total sleep time, and sleep efficiency in nine individuals with Rett syndrome.

In contrast, some reports have been less positive. ${ }^{27}$ The possibility of important side effects has been highlighted by Sheldon, ${ }^{28}$ who reported increased seizure activity in four of six children treated with melatonin. This contrasts with views of Jan et $a l^{15}$ and $\mathrm{O}^{\prime}$ Callaghan et al ${ }^{25}$ that melatonin may have a beneficial effect in suppressing epileptic activity. Nevertheless, history of epilepsy is now often taken clinically to be a contraindication to melatonin therapy. This is unfortunate, given recent evidence that daily melatonin can help reduce epileptic attack frequency in children with sleep disturbances of various aetiologies. ${ }^{29}$

There is therefore a need for further investigation of possible short and long term benefits and adverse effects of melatonin supplementation in young people with sleep problems, associated with severe developmental difficulties of cognitive, social, and communicatory natures, which have not responded to behavioural and social measures. Highly structured double blind cross over methodology is the ideal. This will require well established, reliable, and valid objective and report measures of sleep behaviour, and adequate follow up to ensure long term benefits or otherwise are identified.

\section{CONCLUSIONS}

Evidence from multiple converging sources suggests that oral synthetic melatonin frequently produces marked and welcome improvements in even extremely longstanding and severe sleep disturbance in children and young people with serious and often multiple developmental disabilities. Melatonin can be a highly beneficial, short term, rapid onset, and safe treatment for intractable sleep disturbance in this group. Improvement occurs even when there has been failure to respond to behavioural interventions and when other medications have failed to produce benefits and/or have induced unacceptable side effects. Limited data suggest that therapeutic dose cannot be predicted on grounds of severity and duration of sleep disturbance, severity of intellectual disability, or presence/absence of an autistic spectrum disorder. Habituation can occur, requiring intermittent incremental dosage increases or drug holidays. However, individuals can often be maintained on the same dose for years with no obvious adverse effects. The need for concomitant psychological, behavioural, educational, family, and other social interventions and supports is evident.

The evidence base for the usefulness of melatonin in chronic sleep initiation and maintenance problems in children continues to grow. ${ }^{30}$ However, benefits in other age groups require separate evaluation. One recent study ${ }^{31}$ detected no evidence for melatonin being of benefit in people with dementia who experience serious sleep difficulties.

The mechanisms by which melatonin produces enhanced sleep remain unresolved. It has been suggested, with little supportive evidence, that the action is a simple sedative one. However, this would not explain fully the daytime benefits suggestive of improved sleep quality as well as quantity.
Enhanced central nervous system melatonin receptor response remains a strong probability. This is supported by research suggesting that melatonin supplementation helps correct deficiencies in diurnal secretion cycles. A mild pharmacological antidepressant effect may also contribute, given the anecdotal reports of increased daytime calmness, sociability, and cooperativeness. This notion is supported by the chemical structure of melatonin being similar to that of the mood enhancing neurotransmitter serotonin, and its being part of the same metabolic pathway. Clearly, parents being able to get a good night's sleep is critical as well.

Taken together, reports to date suggest that melatonin is best for sleep induction, less good but still useful for repeated night time waking, and least beneficial for early morning waking. With early morning waking, its importance as a feature of depression requires recognition and systematic evaluation for the presence of this potentially life threatening psychiatric disorder. Diagnosis of depression indicates a need for treatment options consisting of cognitive behavioural psychotherapeutic approaches $^{32}$ and judicious use of modern antidepressants such as selective serotonin reuptake inhibitors. $^{33}$

The historical tendency to prescribe sedating antihistamines for childhood insomnia is steadily diminishing. This is because of high rates of paradoxical excitation with worsening of sleeplessness, particularly in young people with atypical central nervous system development. There are also frequent reports of daytime drowsiness, irritability, headache, dry mouth, behavioural and emotional disinhibition, and cognitive blunting while taking sedating antihistamines at night time for sleeplessness.

The alpha-2 noradrenergic receptor agonist clonidine has a role to play in childhood severe sleeplessness that has failed to respond to behavioural and social measures. It has a well established evidence base in the treatment of hyperkinetic disorder and attention deficit hyperactivity disorder (ADHD), particularly when there is a need to avoid adverse effects on sleep and appetite, in the presence of tic disorder, or when severe epilepsy is a contraindication to psychostimulant medication. ${ }^{34}$ Its safety and efficacy for ADHD in children with intellectual disability has also been confirmed..$^{35}$ Mild sedative properties can be advantageous in facilitating sleep induction and maintenance. Research suggests that clonidine can increase sleep duration in adults who do not have developmental disabilities, albeit at the expense of rapid eye movement sleep duration. ${ }^{36}$ Potential benefits for sleep induction, maintenance, and duration in children and young people with developmental disabilities are only now starting to be explored. ${ }^{37}$

Melatonin seems to have the advantage of being beneficial for sleep induction problems while having few identifiable adverse effects. Recently, it has been proposed that similar benefits may be accrued by using the selective beta-1 adrenergic agonist acebutolol. De Leersnyder et $a l^{38}$ reported their experiences with this melatonin antagonist when given to nine children with Smith-Magenis syndrome who were experiencing the frequently associated severe and intractable sleep difficulties. The authors reported successful suppression of inappropriately high morning melatonin levels. This was associated with improved behaviour and concentration, delayed sleep onset, increased sleep duration, and delayed waking. The authors also made anecdotally based suggestions that beta- 1 adrenergic antagonists improve hyperactivity and enhance cognitive performance. However, evidence for these latter claims is unclear. Nevertheless, results relating to sleep disorders in Smith-Magenis syndrome are persuasive and give hope for individuals with this serious genetic disorder and their families. Replication on larger independent cohorts with double blind, placebo 
controlled randomization design is required to confirm these findings.

In the meantime, melatonin appears to represent an increasingly popular and apparently safe treatment for severe and intractable sleep disturbance in children and young people who have serious developmental difficulties, including those caused genetically.

\section{REFERENCES}

1 Douglas J, Richman N. My child won't sleep. London: Penguin, 1984.

2 Quine L. Sleep problems in children with mental handicap. J Ment Defic Res 1991;35:269-90.

3 Stores G. Sleep studies in children with a mental handicap. J Child Psychol Psychiatry 1992;33:1303-17.

4 Sloper S, Knussen C, Turner S, Cunningham C. Factors related to stress and satisfaction with life in families of children with Down's syndrome. J Child Psychol Psychiatry 1991;32:655-76.

5 Stebbens VA, Dennis J, Samuels MP, Croft CB, Southall DP. Sleep-related upper airway obstruction in a cohort with Down's syndrome. Arch Dis Child $1991 ; 66: 1333-8$

6 Clarke DJ, Waters J, Corbett JA. Adults with Prader-Willi syndrome: abnormalities of sleep and behaviour. J R Soc Med 1989:82:21-4.

7 Vgontzas AN, Kales A, Seip J, Mascari MJ, Bixler EO, Myers DC, VelaBueno AV, Rogan PK. Relationship of sleep abnormalities to patient genotypes in Prader-Willi syndrome. Am J Med Genet 1996;67:78-82.

8 Richdale AL, Cotton S, Hibbit K. Sleep and behaviour disturbance in PraderWilli syndrome: a questionnaire study. J Intellect Disabil Res 1999;43:380-392.

9 Mahowald MW, Iber C, Rosen GM, Krivitt W, Ramsay NKC, Kersey JH, Belani K, Whitley CB. Sleep disordered breathing in the mucopolysaccharidoses. Sleep Res 1989;18:348.

10 Bax MCO, Colville GA. Behaviour in mucopolysaccharide disorders. Arch Dis Child 1995:73:77-81.

11 Gould EL, Loesch DZ, Martin MJ, Hagerman RJ, Armstrong SM, Huggins RM. Melatonin profiles and sleep characteristics in boys with fragile $X$ syndrome: a preliminary study. Am J Med Genet 2000;95:307-15.

12 Vostanis $P$, Harrington R, Prendergast M, Farndon P. Case reports of autism with interstitial deletion of chromosome 17 (p11.2 pl1.2) and monosomy of chromosome 5 (5pter $\rightarrow 5$ p 15.3). Psychiatr Genet 1994;4:109-11.

13 Finucane BM, Konar D, Haas-Givler B, Kurtz MB, Scott Cl. The spasmodic upper-body squeeze: a characteristic behavior in Smith-Magenis Syndrome. Dev Med Child Neurol 1994:36:78-82.

14 Smith AC, Dykens E, Greenberg F. Sleep disturbance in Smith-Magenis syndrome. Am J Med Genet 1998;81:186-91.

15 Jan JE, Espezel H, Appleton RE. The treatment of sleep disorders with melatonin. Dev Med Child Neurol 1994;36:97-107.

16 Miles LEM, Raynal DM, Wilson MA. Blind man living in normal society has circadian rhythms of 24.9 hours. Science 1977;198:421-3.

17 Palm L, Blennow G, Wettenberg L Correction of non-24-hour sleep/wake cycle by melatonin in a blind, retarded boy. Ann Neurol 1991;29:336-9.
18 Weitzman ED, Czeisler CA, Coleman RM, Spielman AJ, Zimmerman JC Dement WC. Delayed sleep phase syndrome, a chronobiological disorder with sleep-onset insomnia. Arch Gen Psychiatry 1981;38:737-46.

19 Arendt J, Aldhous M, English J, Marks V, Arendt JH, Marks M, Folkard S. Some effects of jet-lag and their alleviation by melatonin. Ergonomics 1987;30:1379-93.

20 Macfarlane JG, Cleghorn JM, Brown GM, Streiner DL. The effects of exogenous melatonin on the total sleep time and daytime alertness of chronic insomniacs: a preliminary study. Biol Psychiatry 1991;30:371-6.

21 Dahlitz M, Alvarez B, Vignau J, English J, Arendt J, Parkes JD. Delayed sleep phase syndrome response to melatonin. Lancet 1991;333:1121-4.

22 Jan JE, Espezel H. Melatonin treatment of chronic sleep disorders. Dev Med Child Neurol 1995;37:279-80.

23 Pillar G, Etzioni A, Shahar E, Lavie P. Melatonin treatment in an institutionalised child with psychomotor retardation and an irregular sleepwake pattern. Arch Dis Child 1998;79:63-4.

24 Guilleminault C, McCann CC, Quera-Salva M, Cetel M. Light therapy as treatment of dyschronosis in brain impaired children. Eur $J$ Paediatr 1993:152:754-9.

25 O'Callaghan FJK, Clarke AA, Hancock E, Hunt A, Osborne JP. Use of melatonin to treat sleep disorders in tuberous sclerosis. Dev Med Child Neurol 1999:41:123-6.

26 McArthur AJ, Budden SS. Sleep dysfunction in Rett syndrome: a trial of exogenous melatonin treatment. Dev Med Child Neurol 1998;40:186-92.

27 Camfield P, Gordon K, Dooley J, Camfield C. Melatonin appears ineffective in children with intellectual deficits and fragmented sleep: six " $n$ of $1^{\prime \prime}$ trials. J Child Neurol 1995;11:341-3.

28 Sheldon SH. Pro-convulsant effects of oral melatonin in neurologically disabled children. Lancet 1998;351:1254.

29 Fauteck J, Schmidt H, Lerchl A, Kurlemann G, Wittkowski W. Melatonin in epilepsy: first results of replacement therapy and first clinical results. Biol Signals Receptors 1999:8:105-10.

30 Ivanenko A, Crabtree VM, Tauman R, Gozal D. Melatonin in children and adolescents with insomnia: a retrospective study. Clin Pediat 2003;42:51-58.

31 Serfay M, Kennell-Webb S, Warner J, Blizard R, Raven P. Double blind randomised placebo controlled trial of low dose melatonin for sleep disorders in dementia. Int J Geriatr Psychiatry 2002;17:1120-7.

32 Harrington R, Wood W, Verduyn C. Clinically depressed adolescents. In: Graham P, ed. Cognitive behaviour therapy for children and families. Cambridge: Cambridge University Press, 1998.

33 Findling RL, Feeny NC, Stansbrey RJ, Delporto-Bedoya D, Demeter C. Somatic treatment for depressive illness in children and adolescents. Child Adol Psychiat Clin N Am 2002;11:555-78.

34 Ramchandani $\mathbf{P}$, Joughin C, Zwi M. Attention deficit hyperactivity disorder in children. Clin Evid 2002;7:262-71

35 Agarwal V, Sitholey P, Kumar S, Prasad M. Double-blind, placebo-controlled trial of clonidine in hyperactive children with mental retardation. Ment Retard 2001;39:259-67.

36 Autret A, Minz M, Beillevaire T, Cathala HP, Schmitt $H$. Effect of clonidine on sleep patterns in man. Eur J Clin Pharm 1977;12:319-22.

37 Ingrassia A, Turk J. The use of clonidine for sleep problems in children with neurodevelopmental disorders: a case series. Dev Med Child Neurol 2002;44(Suppl. 92):25.

38 De Leersnyder H, Bresson JL, de Blois MC, Souberbielle JC, Mogenet A, Delhotal-Landes B, Salefranque F, Munnich A. Beta 1-adrenergic antagonists and melatonin reset the clock and restore sleep in a circadian disorder, SmithMagenis syndrome. J Med Genet 2003;40:74-8. 